\title{
Complementary and Alternative Medicine Usage and Yoga Practice in Patients with Metastatic Breast Cancer
}

\author{
Colette Worcester ${ }^{1}$, Chinmay Pandya ${ }^{2 *}$ \\ ${ }^{1}$ University of Kansas School of Medicine, Kansas City, Kansas, United States of America \\ ${ }^{2}$ Dev Sanskriti Vishwavidyalaya, Haridwar, India \\ *Corresponding author email: provc@dsvv.ac.in \\ https://doi.org/10.36018/dsiij.v19i.240
}

\begin{abstract}
Metastatic breast cancer has a poor prognosis, and many patients practice yoga or use other complementary and alternative medicine (CAM) in their healthcare. CAM has risks and benefits, and it will be useful to understand the role of CAM integrated with conventional treatments such as chemotherapy and radiation. Patients with metastatic cancer may respond differently to CAM than those without metastases, and less literature has addressed disease progression in this regard. This short review will attempt to address CAM usage in patients with breast cancer, focused on metastatic disease. The review will address some geographical and cultural differences in CAM usage, benefits of yoga for this patient population to improve quality of life, and highlight study limitations and implementation.
\end{abstract}

Keywords. Metastatic Breast Cancer, Complementary And Alternative Medicine, Yoga, Cultural Differences

\section{INTRODUCTION}

Breast cancer $(\mathrm{BC})$ remains a highly prevalent cancer diagnosed in women, often resulting in a long journey of surgeries and treatments. While therapies have improved over the recent years for primary tumors, metastatic BC (i.e., cancer cells are found in sites distant to the original tumor) still has a poor prognosis. According to the National Cancer Institute's Surveillance, Epidemiology, and End Results (SEER) statistics, the 5-year survival rate for patients with breast cancer that has metastasized is $29 \%$ (1).

Complementary and alternative medicine (CAM) encompasses a wide range of therapies including, but not limited to, dietary supplements, exercise, meditation, prayer, and yoga. Traditional yoga connects physical activity with the mind and spirit. Many cancer patients practice yoga or use other CAM in their healthcare regimen in one of three different approaches. Patients may choose to utilize conventional medicine only, reject conventional approaches and use CAM only, or be somewhere in the middle with both conventional and CAM medicine utilization. The patients' rational for integrating CAM with conventional chemotherapy and radiation should be understood to explore risks and benefits and tailor individualized treatment plans. 
Compared to patients without metastasis, patients with metastatic breast cancer may respond differently to CAM. This short review will attempt to address CAM usage in patients with breast cancer, focused on metastatic disease. The review will address some geographical and cultural differences, benefits of yoga for this patient population, and highlight limitations in current studies and implementation.

\section{USAGE OF CAM BY PATIENTS WITH METASTATIC BREAST CANCER}

\section{CAM usage}

Delineation of metastatic disease is not always noted in CAM studies, but breast cancer patients use CAM. A 2010 systematic review revealed that patients with breast cancer were among the highest CAM users compared to other cancer types (2). Interestingly, further studies looking at CAM usage and the rationale to engage in these practices have led to diverse findings, suggesting that geographical location, cultural background, and political systems must be included and acknowledged in any study of CAM.

In a study with Chinese breast cancer patients, nearly all the participants reported the use of CAM (3), with walking being a popular CAM utilized. In a questionnaire for a sub-Himalayan city in India, CAM usage was highest in patients with breast cancer compared to other cancers, while the predominant CAM noted was yoga (4). Likely the difference in culture and location, reporting methods, criteria of CAM, or criteria of breast cancer stage may contribute to the vast differences in the usage type and percent of patients. It may be possible that another factor for the differences is the economic model of each country, as an economic ideology may affect whether CAM is approved centrally by the government or whether individuals have free choice.
Indeed, CAM usage is also different in the United States. Yoga implementation is on the rise in the US, with greater than $13 \%$ of the general population practicing in 2017, though it varied even with geographical location within the US (5). Similarly, 1 in 4 breast cancer patients in the US reported using mindfulness, primarily spiritual and yoga practices (6).

While much more is described for $\mathrm{BC}$ patients without subgrouping for metastasis, patients with metastatic BC do also use CAM. In Brazil, about half of patients with metastatic breast cancer reported CAM use, the most popular being dietary supplements (7). According to a survey of breast cancer patients in Indonesia, CAM usage was significantly higher with distant metastases, though the type of CAM was unspecified (8). Thus, studies highlight that metastatic disease may be a factor for CAM.

\section{Rationale for using CAM}

Knowing that the prevalence of CAM usage differs, it is also important to know the reasons why patients choose CAM. According to a 2010 systemic review, patients that were younger and had higher education, income, and access to health insurance were more likely to use CAM (2). Contrarily, a 2016 survey showed BC patients in Indonesia who had lower education levels and lower income more likely to use CAM (8). The contradiction may again be a highlight of different patient populations.

Additionally, BC patients who only somewhat trusted their physicians, or had been diagnosed with breast cancer more than 12 months ago were more likely to use CAM (8). While differences in geographic location and healthcare and insurance regulations exist, physician mistrust is significant even amongst the younger, well-educated cancer patients in Texas, US (9). Trust is crucial to the physician-patient relationship for patients to adhere 
to the recommended plan. It would be interesting to correlate CAM usage with physician distrust by nation, and to examine whether the country has public insurance, private insurance, or both. Further studies to understand the specific patient population, depending on location, culture, and educational and healthcare background are needed when determining the CAM usage, rather than generalizing findings.

Oncologists and other providers may not be aware of their patients' CAM usage, and thus cannot monitor the safety. Samuels et al. (2017) reports that patients in Israel with metastatic breast cancer were more likely than not to use herbal medicine, but the vast majority of those surveyed did not have their CAM usage monitored or disclosed to their health care providers. Furthermore, almost half of the patients using herbal medicine used them as cancer or immune treatments (10). The study showed an important role for an integrative physician to advise patients about CAM to reduce side effects or improve quality of life, rather than using CAM for curative therapy. Further, integrative physicians can monitor for adverse drug-herb interactions, as some patients in the study were recommended either to stop or to start an herbal supplement based on their therapeutical regimen and healthcare goals (10).

\section{Summary of CAM Usage}

Differences of CAM acceptance and usage, including yoga, differs in geographical location, and it remains to be seen if the differences occur with respect to the following aspects:

- Economic statuses and models

- Governmental legislation and regulations

- Patient perceptions and knowledge about CAM

- Physician perceptions and knowledge about CAM

- Perceptions about physicians and available healthcare

- Educational statuses of the population
- Systemic healthcare and insurance structures

There are challenges to create evidence-based medical recommendations about CAM, including yoga, for patients of a non-homogenous population.

\section{HEALTH EFFECTS OF YOGA FOR PATIENTS WITH METASTATIC BREAST CANCER}

\section{Yoga benefits in BC patients with conventional therapy}

Conventional therapies for BC patients include chemotherapy and radiation, both of which can decrease a patient's quality of life through side effects. CAM may have a role in improving quality of life concurrently with conventional medicine. After receiving radiation therapy, $\mathrm{BC}$ patients in Germany adapted their CAM usage and begin to practice yoga and/or relaxation methods more than prior to radiation (11). The CAM usage did not depend on additional cancer therapies aside from the radiation (11).

A 2017 systematic review showing improved quality of life in breast cancer patients who practice yoga while undergoing radiation has been previously described by Galliford and colleagues (12). Some of the breast cancer-related symptoms that have been relieved by yoga include stress, sleep, pain, and fatigue (13-17). A study stratified the state of mindfulness and saw that an increased perception of mindfulness in patients with metastatic BC decreased their perception of pain and fatigue (13). Breast cancer patients on chemotherapy in India had improved fatigue and insomnia when trained to practice yoga twice daily (17). Many similar studies have shown that yoga increases quality of life, and a Cochrane systematic review compared yoga intervention versus no intervention in breast cancer patients. While the evidence was lacking to factor in metastatic disease, the review showed yoga improved quality of life 
attributes in those with non-metastatic disease (18). However, this review did not find any differences between yoga versus exercise, giving some credence that movement in general may alleviate symptoms or help in improving quality of life.

\section{Yoga benefits in metastatic BC patients}

Patients with cancer that has metastasized have a worse prognosis and may require a milieu of conventional therapies, and it is important to understand whether metastatic disease plays a role in the amount of fatigue improvement gained with yoga. Specifically for metastatic disease, patients with metastatic breast cancer undergoing radiotherapy and integrated yoga intervention for three months had improved symptoms in fatigue severity and frequency when compared to patients receiving supportive care (16). The amount or duration of yoga may also be important to consider. In one 9-week study, patients with metastatic breast cancer who practiced more yoga had lower levels of pain than when the same patients spent less time practicing yoga (19).

Because cancer patients often experience stress and stress is related to negative health impacts, alleviating stress and such related symptoms would be beneficial. One three month intervention in patients with metastatic breast cancer showed improvements in sleep patterns and decreased morning cortisol levels after yoga compared to supportive therapy (20). Cortisol is an important maker of stress hormones, but it is not clear whether its decrease was directly due to the yoga or indirectly, as cortisol is related to the circadian rhythm and sleep patterns. Further studies are needed to determine the role of movement compared to yoga and to elucidate whether yoga improves the quality of life in breast cancer patients with metastatic disease.

\section{Yoga benefits in the long-term}

While most studies correlate therapeutic strategies with symptoms during or just post treatment, few have examined long-term outcomes. One exception is a Chinese survey-based study that noted the majority of breast cancer survivors (at least 5 years post-diagnosis) had adaptive coping strategies, though almost $15 \%$ of patients had signs of maladaptive coping or distress. The survey allowed researchers to use modeling to predict anxiety. The researchers claimed that clinical stage was not a predictor of coping, though the study was not focused on demographic factors (21).

Yoga may be beneficial in the long-term coping, as well. One case-control study in India reported that having yoga practice in the past correlated with decreased anxiety and depression in early breast cancer survivors who had finished their treatments at least six months prior (22). Therefore, further studies could be implemented to address the longterm quality of life and psychosocial factors for women with metastatic breast cancer. Particularly, it may be beneficial to address yoga and other CAM use long-term, especially long after conventional chemotherapy and radiation have been completed.

\section{Summary for yoga health effects}

Research for breast cancer patients specifically with metastatic disease should further elucidate:

- Differences in yoga effects for breast cancer stage

- Long term studies to study the impacts of yoga on stress and quality of life

- Yoga compared to other physical movement and meditation

\section{STUDY DESIGNS FOR YOGA IN PATIENTS WITH METASTATIC BREAST CANCER}

Designing a randomized control trial to study the effects of yoga is challenging. By the nature of the study, it is impossible to blind participants or eliminate all biases. Many studies have compared 
yoga to no intervention, though it is possible that having any form of therapy may benefit patients. To take that notion into account, other studies have compared yoga to a support group intervention. Other considerations include needing approval from ethics committees for a randomized control trial involving yoga or other CAM in patients. As addressed earlier, the acceptability and cultural practices vary in geographic region, and must be considered when designing a study and encouraging adherence to the plan. In India, the government recognizes a CAM system called Ayurveda, Yoga and Naturopathy, Unani, Siddha, Sowa-Rigpa and Homeopathy (AYUSH), and approximately $7 \%$ of healthcare patients, from both high and low-income groups, also utilize treatments related to AYUSH (23). While CAM has been widely accepted in regions such as India or China for generations, a legislative framework for implementing CAM lacks in other countries, such as the US. Nevertheless, policies and programs are being implemented in the US for schoolchildren to gain awareness of yoga (24). It may be possible that more awareness may lead to increased acceptance for integrative medicine.

Policies to regulate yoga are important from a public acceptance view, but they are also needed from a research perspective. In a 2019 review, Goyal discussed the lack of standardization in yoga interventions in studies. Some studies utilize selfpractice, while others utilize group instruction. Indeed, the frequency, duration, and practices differ from study to study. Yoga can be physically demanding, and the author highlights the need to standardize yoga for patient safety and recommends not using yoga in medical research unless it is clearly defined and standardized (25). Indeed, another study reported yoga injuries from a survey of people practicing yoga in Germany, with injuries more common in those practicing without supervision (26). However, the injuries from yoga were not more common than from other forms of exercise (26). Such concerns should be addressed to provide evidence-based recommendations about yoga.

Another challenge with studies involving yoga is feasibility. Some studies showed difficulty in retaining study participants $(14,27)$. Because of scheduling constraints on breast cancer patients undergoing chemotherapy and radiation treatments, feasibility in yoga interventions are vital. A pilot program found a virtual platform to be a feasible option, though flexibility in patients' schedules and varying symptoms need improvement (28).

\section{Summary of yoga study designs}

Study designs for yoga are challenging with respect to culture, control groups, standardization, and participant retention. Features to be further parsed out include:

- How the country's policies affect studies involving yoga

- How yoga can be standardized in studies

- Adverse or safety concerns of yoga and CAM

○ The best platform for delivering yoga

Good study design will inform evidence-based medicine for physicians to advise breast cancer patients on the best approaches for their health.

\section{CONCLUSION}

In conclusion, many types of CAM, including yoga, are integral for many patients with metastatic breast cancer, either following diagnosis, during treatment, or even after conventional treatments have been completed. CAM varies with culture and geographic region, and must be further understood. Notably, yoga has been shown to improve quality of life, such as fatigue and pain, in patients with metastatic breast cancer. 
Yoga intervention studies are not always feasible for patients' schedules in concurrence with radiation and chemotherapy time and symptom constraints. In addition, yoga is not standardized across studies, and healthcare providers and patients must be aware of the potential benefits in yoga practice as well as potential injuries, as when implementing any form of physical activity. Further studies to standardize yoga practice in medical research and observe long-term effects are also needed.

The needs of patients with metastatic breast cancer may differ from those with early-stage breast cancer, and literature focused on CAM with breast cancer have not often addressed the effect of metastatic disease, which needs to be further studied. In the face of late-stage breast cancer treatments, healthcare providers must aid patients in making decisions with an individualized approach to live the best life they can. Further studies are warranted to parse out when yoga can be beneficially synergistic with conventional therapies.

Sponsoring information: The Virtual Mojo Summer 2021 Internship and Indian Cultural Immersion; Office of International Programs, University of Kansas Medical Center, Kansas City, KS, USA

Competing interests: None declared.

Patient consent for publication: Not required.

\section{REFERENCES}

1. National Cancer Institute. Cancer Database 2011-2018 [Internet]. Surveillance, Epidemiology, and End Results (SEER) $\quad$ Program. 2018. https://seer.cancer.gov/statfacts/html/breast.html

2. Wanchai A, Armer JM, Stewart BR. Complementary and alternative medicine use among women with breast cancer: a systematic review. Clin J Oncol Nurs. 2010 Aug;14(4):E45-55.

https://doi.org/10.1188/10.CJON.E45-E55

3. Chen Z, Gu K, Zheng Y, Zheng W, Lu W, Shu XO. The use of complementary and alternative medicine among Chinese women with breast cancer. J Altern
Complement Med. 2008 Oct;14(8):1049-55. https://doi.org/10.1089/acm.2008.0039

4. Pandey L, Pasricha R, Joseph D, Ahuja R, Yanthan Y, Garg PK, et al. Use of complementary and alternative medicine among patients with cancer in a subHimalayan state in India: An exploratory study. J Ayurveda Integr Med. 2021;12(1):126-30. https://doi.org/10.1016/j.jaim.2021.01.001

5. Zhang Y, Lauche R, Cramer H, Munk N, Dennis JA. Increasing Trend of Yoga Practice Among U.S. Adults from 2002 to 2017. J Altern Complement Med. 2021 Jun;27(9):778-85.

https://doi.org/10.1089/acm.2020.0506

6. Voiß P, Höxtermann MD, Dobos G, Cramer H. Mindbody medicine use by women diagnosed with breast cancer: results of a nationally representative survey. Support care cancer Off J Multinatl Assoc Support Care Cancer. 2020 Mar;28(3):1077-82. https://doi.org/10.1007/s00520-019-04914-x

7. Alfano ACC, Paiva CE, Rugno FC, da Silva RH, Paiva BSR. Biologically based therapies are commonly selfprescribed by Brazilian women for the treatment of advanced breast cancer or its symptoms. Support care cancer Off J Multinatl Assoc Support Care Cancer. 2014 May;22(5):1303-11. https://doi.org/10.1007/s00520-013-2087-X

8. Azhar Y, Achmad D, Lukman K, Hilmanto D, Aryandono T. Predictors of Complementary and Alternative Medicine Use by Breast Cancer Patients in Bandung, Indonesia. Asian Pac J Cancer Prev. 2016;17(4):2115-8. https://doi.org/10.7314/APJCP.2016.17.4.2115

9. Grant S, Liao K, Miller C, Peterson S, Elting L, Guadagnolo BA. Lower Levels of Trust in the Medical Profession Among White, Younger, and Moreeducated Individuals With Cancer. Am J Clin Oncol. 2021 Apr;44(4):150-7. https://doi.org/10.1097/COC.0000000000000771

10. Samuels N, Ben-Arye E, Maimon Y, Berger R. Unmonitored use of herbal medicine by patients with breast cancer: reframing expectations. J Cancer Res Clin Oncol. 2017 Nov;143(11):2267-73. https://doi.org/10.1007/s00432-017-2471-x

11. Männle H, Momm F, Hübner J, Münstedt K. Do breast cancer patients adapt CAM methods according to the therapeutic situation? Complement Ther Clin Pract. $2021 \quad$ May;43:101305. https://doi.org/10.1016/j.ctcp.2021.101305

12. Galliford M, Robinson S, Bridge P, Carmichael M. Salute to the sun: a new dawn in yoga therapy for breast cancer. J Med Radiat Sci. 2017 Sep;64(3):232-8. https://doi.org/10.1002/jmrs.218 
13. Zimmaro LA, Carson JW, Olsen MK, Sanders LL, Keefe FJ, Porter LS. Greater mindfulness associated with lower pain, fatigue, and psychological distress in women with metastatic breast cancer. Psychooncology. 2020 Feb;29(2):263-70. https://doi.org/10.1002/pon.5223

14. Porter LS, Carson JW, Olsen M, Carson KM, Sanders $\mathrm{L}$, Jones L, et al. Feasibility of a mindful yoga program for women with metastatic breast cancer: results of a randomized pilot study. Support care cancer Off J Multinatl Assoc Support Care Cancer. 2019 Nov;27(11):4307-16. https://doi.org/10.1007/s00520019-04710-7

15. Vadiraja HS, Rao RM, Nagarathna R, Nagendra HR, Patil S, Diwakar RB, et al. Effects of Yoga in Managing Fatigue in Breast Cancer Patients: A Randomized Controlled Trial. Indian J Palliat Care. 2017;23(3):247-52. https://doi.org/10.4103/IJPC.IJPC_95_17

16. Vadiraja HS, Rao MR, Nagarathna R, Nagendra HR, Rekha M, Vanitha N, et al. Effects of yoga program on quality of life and affect in early breast cancer patients undergoing adjuvant radiotherapy: a randomized controlled trial. Complement Ther Med. 2009;17(56):274-80. https://doi.org/10.1016/j.ctim.2009.06.004

17. Prakash K, Saini SK, Pugazhendi S. Effectiveness of Yoga on Quality of Life of Breast Cancer Patients Undergoing Chemotherapy: A Randomized Clinical Controlled Study. Indian J Palliat Care. 2020;26(3):323-31.

https://doi.org/10.4103/IJPC.IJPC_192_19

18. Cramer H, Lauche R, Klose P, Lange S, Langhorst J, Dobos GJ. Yoga for improving health-related quality of life, mental health and cancer-related symptoms in women diagnosed with breast cancer. Cochrane database Syst Rev. 2017 Jan;1(1):CD010802. https://doi.org/10.1002/14651858.CD010802.pub2

19. Carson JW, Carson KM, Olsen M, Sanders L, Westbrook K, Keefe FJ, et al. Yoga Practice Predicts Improvements in Day-to-Day Pain in Women With Metastatic Breast Cancer. J Pain Symptom Manage. 2021 Jun;61(6):1227-33. https://doi.org/10.1016/j.jpainsymman.2020.10.009

20. Rao RM, Vadiraja HS, Nagaratna R, Gopinath KS, Patil S, Diwakar RB, et al. Effect of Yoga on Sleep Quality and Neuroendocrine Immune Response in Metastatic Breast Cancer Patients. Indian J Palliat Care. 2017;23(3):253-60. https://doi.org/10.4103/IJPC.IJPC_102_17

21. Cheng C-T, Ho SMY, Lai Y, Zhang Q, Wang G-L. Coping profiles predict long-term anxiety trajectory in breast cancer survivors. Support care cancer Off J Multinatl Assoc Support Care Cancer. 2021 Jul;29(7):4045-53. https://doi.org/10.1007/s00520$\underline{020-05936-6}$
22. Amritanshu RR, Rao RM, Nagaratna R, Veldore VH, Usha Rani MU, Gopinath KS, et al. Effect of Longterm Yoga Practice on Psychological outcomes in Breast Cancer Survivors. Indian $J$ Palliat Care. 2017;23(3):231-6. https://doi.org/10.4103/IJPC.IJPC $93 \_17$

23. Rudra S, Kalra A, Kumar A, Joe W. Utilization of alternative systems of medicine as health care services in India: Evidence on AYUSH care from NSS 2014. PLoS One. 2017;12(5):e0176916. https://doi.org/10.1371/journal.pone.0176916

24. Butzer B, Ebert M, Telles S, Khalsa SBS. School-based Yoga Programs in the United States: A Survey. Adv Mind Body Med. 2015;29(4):18-26.

25. Goyal A. Call for global standards in clinical yoga trials. Yoga Mimamsa [Internet]. 2019 Jul 1;51(2):637. https://doi.org/10.4103/ym.ym_12_19

26. Cramer H, Quinker D, Schumann D, Wardle J, Dobos G, Lauche R. Adverse effects of yoga: a national crosssectional survey. BMC Complement Altern Med. 2019 Jul;19(1):190. https://doi.org/10.1186/s12906-019$\underline{2612-7}$

27. Carson JW, Carson KM, Olsen MK, Sanders L, Porter LS. Mindful Yoga for women with metastatic breast cancer: design of a randomized controlled trial. BMC Complement Altern Med. 2017 Mar;17(1):153. https://doi.org/10.1186/s12906-017-1672-9

28. Addington EL, Sohl SJ, Tooze JA, Danhauer SC. Convenient and Live Movement (CALM) for women undergoing breast cancer treatment: Challenges and recommendations for internet-based yoga research. Complement Ther Med. 2018 Apr;37:77-9. https://doi.org/10.1016/j.ctim.2018.02.001 